\title{
Evaluation of Noncovalent Interactions between Peptides and Polyether Compounds via Energy-Variable Collisionally Activated Dissociation
}

\author{
Matthew C. Crowe and Jennifer S. Brodbelt \\ Department of Chemistry and Biochemistry, University of Texas, Austin, Texas, USA
}

\begin{abstract}
Energy-variable collisionally activated dissociation (CAD) was used to analyze noncovalent interactions of protonated peptide/polyether complexes in a quadrupole ion trap complexes were formed with a series of four polyether host molecules and thirteen peptide molecules. Comparison of dissociation thresholds revealed correlations between the gas-phase basicities of the peptides and polyether molecules and the onset of dissociation. The dissociation thresholds of complexes containing the tripeptides or pentapeptides were inversely proportional to the gas-phase basicities of the sites of protonation of the peptides. Intramolecular hydrogen bonding of the pentapeptides affected the observed dissociation thresholds as well. The dissociation thresholds also scaled proportionally to the gas-phase basicities of the polyethers in the complexes, and the importance of the conformational flexibility of the polyether ligand was confirmed for one of the histidine-containing tripeptide complexes. (J Am Soc Mass Spectrom 2003, 14, 1148-1157) (c) 2003 American Society for Mass Spectrometry
\end{abstract}

$\mathrm{T}$ The advent of "soft" ionization sources such as electrospray ionization (ESI) [1] and matrix assisted laser desorption ionization (MALDI) [2] has allowed observation of intact noncovalent complexes in the gas phase [3-7]. Since noncovalent interactions are crucial to biological systems, the ability to transport these types of complexes from the solution phase to the gas phase for subsequent characterization by mass spectrometry enables us to probe supramolecular interactions in a solvent-free environment and begin to develop an understanding of the intrinsic nature of noncovalent interactions, such as hydrogen bonding. Noncovalent interactions are important in the formation of higher order structures of proteins as well as in the interactions of proteins with other biological molecules in solution, and there is great interest in evaluating the survival of such interactions in the gas phase and understanding the extent to which biological complexes in the gas phase relate to the native ones in solution. Studying how different variables affect the strengths of noncovalent interactions in relatively simple systems is the first step in providing information that may be useful in assessing the nature of noncovalent interactions in larger, biologically relevant com-

Published online August 11, 2003

Address reprint requests to Dr. J. S. Brodbelt, Department of Chemistry and Biochemistry, 1 University Station A5300, Austin, TX 78712-0165, USA. E-mail: jbrodbelt@mail.utexas.edu plexes. For example, synthetic ligands can serve as models for biological molecules; crown ethers first described by Pederson in 1967 [8] have been frequently studied both in solution [9] and in the gas phase [10-38] and mimic simple biological molecules with hydrophilic binding cavities [39]. In addition, crown ethers can be used to model charge solvation processes because of their polydentate nature and affinity for cations.

Crown ethers have been shown to form stable gasphase complexes with peptide molecules containing basic sites, either at the N-terminus or at a basic amino acid side chain [40-42]. Some work with peptide/ crown ether complexes has been focused on charge state determination [40], in which the addition of 18crown- 6 to a peptide ion facilitated determination of the charge state using constant neutral loss scans. Beauchamp and co-workers have also undertaken amino acid side chain-specific recognition with several crown ether hosts $[41,42]$. These studies suggested that 18-crown- 6 preferentially binds to the side chain of lysine over other available basic sites [41], and that larger hosts like dibenzo-30-crown-10 and 27-crown-9 bind strongly to the guanidinium side chain of an arginine residue [42]. Beauchamp et al. also hypothesized that the number of specific basic amino acids present in a peptide molecule could be quantified using these types of noncovalent interactions [42]. The solvation of a protonated amine site in a peptide by a crown 


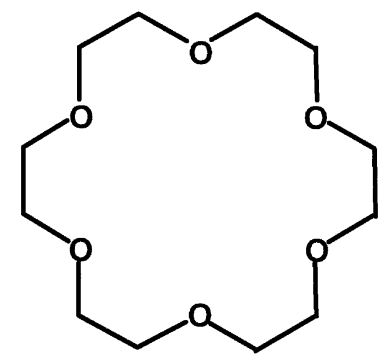

18-Crown-6 (217.4)

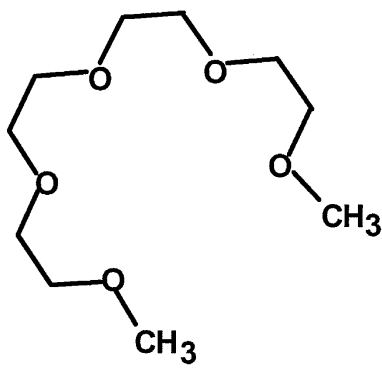

Tetraglyme (214.6)

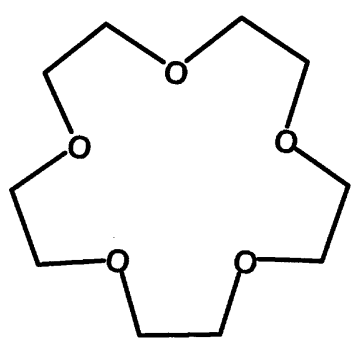

15-Crown-5 (215.0)

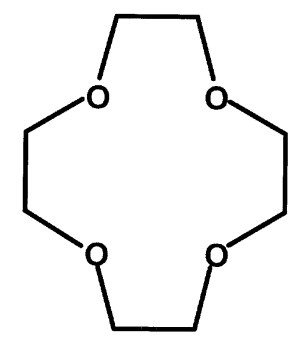

12-Crown-4 (212.8)
Figure 1. Structures of hosts (gas-phase basicity in $\mathrm{kcal} / \mathrm{mol}$ [44]).

ether may disrupt some of the intramolecular hydrogen bonds in the peptide, and thus this artificial solvation process may be envisioned as a way to selectively probe aspects of secondary structure in peptides (or proteins).

In the present study, we have compared the strengths of the noncovalent interactions between various protonated peptide molecules (KGG, GKG, GGK, HGG, GHG, GGH, GGG, YGGFL, YGRFL, AAAAA, KAAAA, RAAAA, AAAAR) and a set of polyethers (18-crown-6, 15-crown-5, 12-crown-5, and the acyclic host tetraglyme) (Figure 1) based on energy-variable collisionally activated dissociation in a quadrupole ion trap mass spectrometer. For peptides without basic residues, protonation occurs at the N-terminus in gasphase ions, whereas the most favorable site of protonation on peptides containing a basic residue is the basic side chain functional group [43]. In the present work, energy-variable CAD measurements are used to compare the relative dissociation energies of the noncovalent peptide/polyether complexes. As shown herein, the relative dissociation energies of noncovalent complexes containing different isomeric peptides solvated by the same polyether provide a sensitive way to assess subtle differences in the factors that influence the strengths of noncovalent interactions.

Polyether complexes involving three series of peptides were examined in this study. The tripeptides (KGG, GKG, GGK, HGG, GHG, GGH, and GGG) were selected to allow observation of how changes in both basic residue position and type affect binding strength; GGG allows comparison of complexes involving poly-

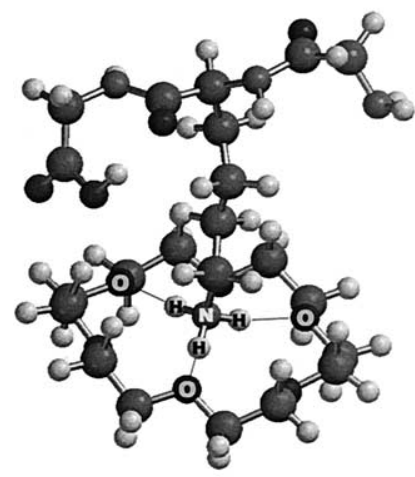

Side

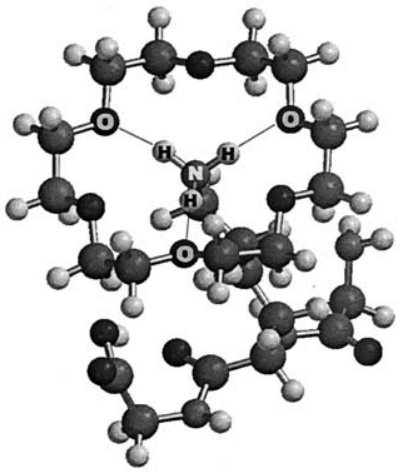

Bottom
Figure 2. Molecular modeling of the protonated GKG/18crown-6 complex.

ether attachment at the protonated N-terminus versus the protonated basic side chain. The penta-alanine peptides (AAAAA, KAAAA, RAAAA, AAAAR) demonstrate how the presence, absence, type, and position of a basic residue affect the dissociation thresholds for larger peptides. The higher degree of intramolecular hydrogen bonding in these peptides affects the binding with the crown ether hosts. The leucine enkephalin pentapeptides (YGGFL, YGRFL) allow the comparison of the binding strength of the host molecule to the protonated $\mathrm{N}$-terminus versus the protonated arginine side chain. The latter two peptides have the potential for considerably more intramolecular hydrogen bonding than any of the other peptides.

In order to assist in conceptualization of these complexes, the Spartan 2002 software suite was utilized for molecular mechanics and semi-empirical calculations at the PM3 level.

\section{Experimental}

Five peptides (GGG, GGH, GHG, AAAAA, YGGFL) were obtained from Sigma Chemical (St. Louis, MO). Four (HGG, GKG, GGK, KGG) were obtained from BACHEM (King of Prussia, PA). The remaining peptides (RAAAA, KAAAA, AAAAR, YGRFL) were synthesized at the Protein Microanalysis Facility at the University of Texas at Austin. The ligands used in these studies (18-crown-6, 15-crown-5, 12-crown-4, and tetraglyme) were all purchased from Aldrich (St. Louis, MO).

All solutions were prepared in $99 \%$ methanol/1\% acetic acid, and the concentration of each species (peptides and ligands) was $1.0 \times 10^{-5} \mathrm{M}$. For each threshold dissociation experiment, a solution consisting of a single peptide and a single polyether ligand at equimolar concentrations was analyzed.

All experiments were performed on a Finnigan LCQDuo using the Xcalibur (Finnigan, San Jose, CA) software package and an electrospray source. The ion trap was operated at a base pressure of nominally $5 \times 10^{-6}$ torr with helium. For ESI experiments, the sample flow rate was $3 \mu \mathrm{L} / \mathrm{min}$, and the pressure in the analyzer 


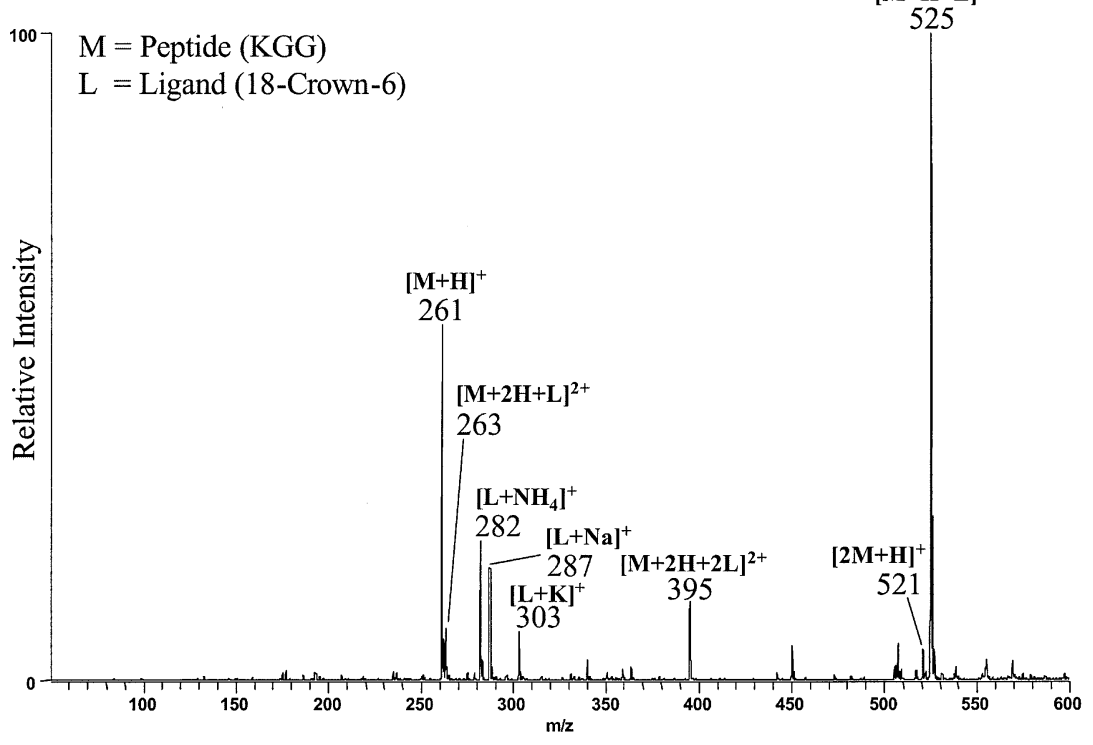

Figure 3. ESI-Mass spectrum of KGG/18-crown-6 solution.

region was then nominally $1 \times 10^{-5}$ torr. Ionization and trapping conditions were optimized once for each of the sets of peptides (lysine tripeptides, histidine tripeptides, leucine enkephalin-type pentapeptides, pentaalanine-type peptides) with each individual ligand. Once instrumental parameters were optimized for one peptide in one of the aforementioned sets for a particular ligand, they were not retuned for subsequent peptides in that set. All experiments were run in triplicate.

All modeling studies were performed using the Spartan 2002 software suite. Conformational searches were performed using molecular mechanics, followed by energy minimization of the most favorable structures at the PM3 level.

\section{Results and Discussion}

\section{Overview}

Protonated peptides form stable noncovalent complexes with polyether molecules; an example of the protonated GKG/18-crown-6 complex is modeled in Figure 2. The electron-rich polyethers "solvate" the protonated amine groups of the peptides, and thus the resulting complexes provide a simple model of charge

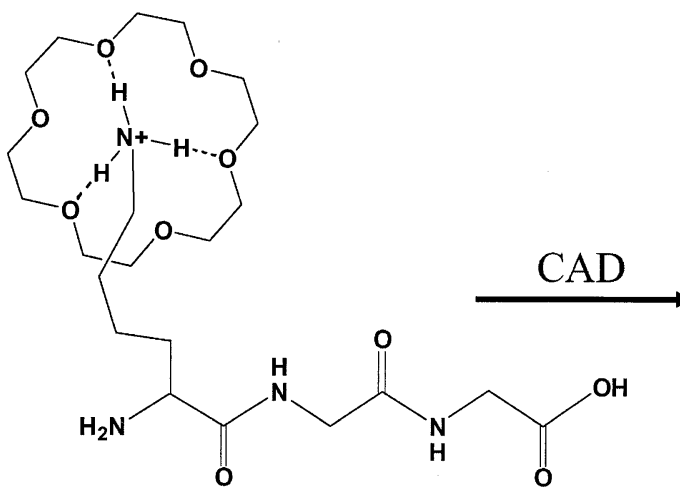

$[\mathrm{KGG}+\mathrm{H}+18 \mathrm{C} 6]^{+}$

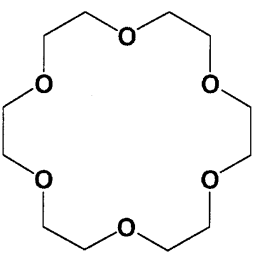

$18 \mathrm{C} 6$<smiles>NCCCCC(N)C(=O)NCC(=O)NCC(=O)O</smiles>

$[\mathrm{KGG}+\mathrm{H}]^{+}$

Scheme 1. Dissociation of the protonated KGG/18-crown-6 complex. 


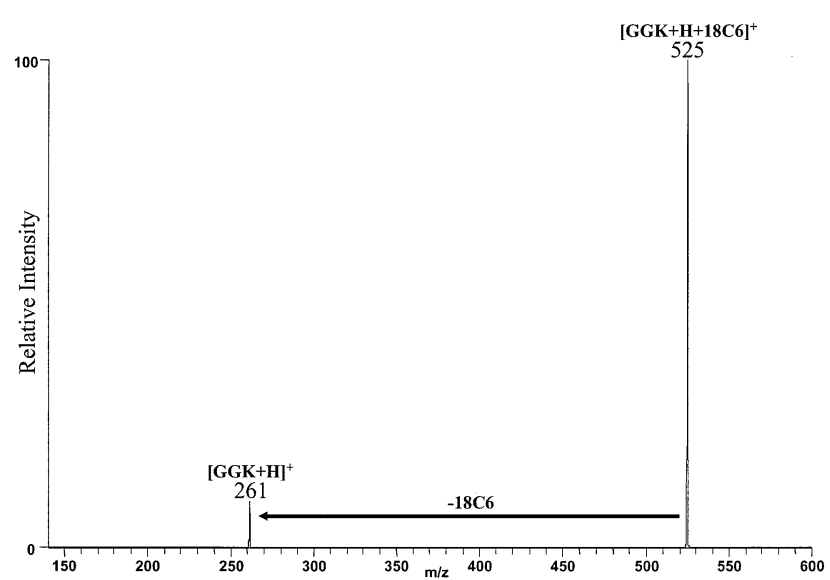

Figure 4. Threshold dissociation of the protonated GGK/18crown-6 complex.

site solvation via hydrogen bond formation in biological complexes. In the peptides containing amino acids with basic side chains (histidine $[\mathrm{H}]$, lysine $[\mathrm{K}]$, arginine $[R])$, the crown ether most likely binds to the protonated side chain, in agreement with studies by Julian and Beauchamp [41]. In the peptides that contain no basic site other than the $\mathrm{N}$-terminus, the crown ethers bind to this protonated primary amine. We are interested in the relative strengths of the hydrogen bonding interactions between the polyether and the protonated peptide as well as the factors affecting the binding strength, as determined by threshold dissociation experiments, in addition to the implications that these results may have for larger noncovalently bound species. An example of the ESI-mass spectrum obtained upon spraying the KGG/18-crown-6 solution is shown in Figure 3. The base peak is the $\left[\mathrm{KGG}+\mathrm{H}^{+}+18\right.$-crown-6] complex.

After transfer to the gas phase by ESI, the parent complex ([peptide $+\mathrm{H}^{+}+$polyether]) was isolated with an isolation window width sufficiently narrow to exclude all but the ${ }^{12} \mathrm{C}$ isotope. Following isolation, the complex was subject to collisional activation $\left(\mathrm{q}_{\mathrm{z}}=\right.$
Table 1. Experimental gas-phase basicities for glycine, histidine, and lysine tripeptides

\begin{tabular}{lc}
\hline Peptide & $\begin{array}{c}\text { Gas-phase basicity }(\mathrm{kcal} / \mathrm{mol}) \text { (determined via the } \\
\text { deprotonation reaction method) }\end{array}$ \\
\hline \hline GGG & $219.1^{\mathrm{a}}$ \\
KGG & $234.3 \pm 3.2^{\mathrm{b}}$ \\
GKG & $228.3 \pm 3.3^{\mathrm{b}}$ \\
GGK & $234.3 \pm 3.2^{\mathrm{b}}$ \\
HGG & $231.3 \pm 3.8^{\mathrm{b}}$ \\
GHG & $228.3 \pm 3.3^{\mathrm{b}}$ \\
GGH & $231.3 \pm 3.8^{\mathrm{b}}$
\end{tabular}

aFrom Reference [44].

bFrom Reference [43] corrected for the current NIST gas-phase basicity scale, Reference [44].

0.250 , activation time $=30 \mathrm{~ms}$ ) at $\mathrm{CAD}$ voltages increasing in integrals of $1 \%$ (relative to the maximum CAD voltage of the ion trap instrument). For each of the complexes, the dissociation resulted in the loss of the neutral crown ether molecule. This dissociation pathway is represented in Scheme 1. The collision voltage required to convert $10 \%$ of the initial parent ion intensity of the complex to the protonated peptide (as illustrated in the CAD spectrum in Figure 4) was tabulated as the threshold voltage. A set of representative dissociation threshold curves for three protonated tripeptide/18-crown-6 complexes (plotted as the percentage of the total ion intensity represented by the fragment ion current versus the CAD voltage applied) with the associated error bars is illustrated in Figure 5. These were obtained via triplicate measurements for each complex, followed by averaging and calculation of the standard deviation. In one case involving the dissociation of the protonated GGG/18-crown-6 complex, the parent complex dissociated to form both protonated 18-crown-6 and GGG due to the relatively low gasphase basicity of tri-glycine, which is comparable to that of 18-crown-6 (see Table 1 and Figure 1). Threshold dissociation data was not recorded for this complex

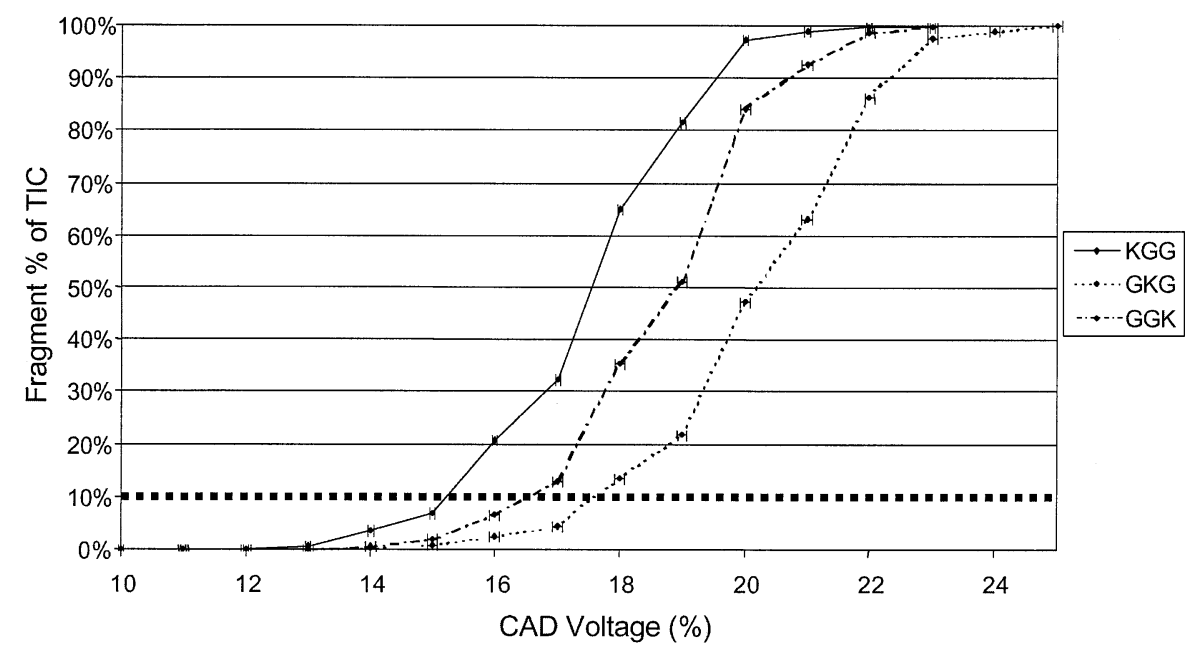

Figure 5. Energy-variable dissociation of protonated lysine tripeptide/18-crown-6 complexes. 
Table 2. Dissociation threshold for protonated tripeptide complexes ${ }^{\mathrm{a}}$

\begin{tabular}{lcccc}
\hline Peptide & $18 \mathrm{C} 6$ & $15 \mathrm{C} 5$ & Tetraglyme & $12 \mathrm{C} 4$ \\
\hline \hline KGG & $15.20 \pm 0.04$ & $11.92 \pm 0.03$ & $11.31 \pm 0.09$ & $9.1 \pm 0.1$ \\
& $(15.20 \pm 0.04)$ & $(13.08 \pm 0.03)$ & $(12.01 \pm 0.09)$ & $(1.1 \pm 0.1)$ \\
GKG & $17.60 \pm 0.08$ & $14.7 \pm 0.3$ & $13.9 \pm 0.2$ & $11.6 \pm 0.2$ \\
& $(17.60 \pm 0.08)$ & $(16.1 \pm 0.3)$ & $(14.8 \pm 0.2)$ & $(14.0 \pm 0.2)$ \\
GGK & $16.52 \pm 0.08$ & $13.08 \pm 0.07$ & $12.2 \pm 0.2$ & $10.1 \pm 0.1$ \\
& $(16.52 \pm 0.08)$ & $(14.35 \pm 0.07)$ & $13.0 \pm 0.2)$ & $(13.3 \pm 0.1)$ \\
HGG & $14.9 \pm 0.2$ & $12.5 \pm 0.3$ & $(13.8 \pm 0.3)$ & $10.3 \pm 0.1$ \\
& $(15.7 \pm 0.2)$ & $(14.5 \pm 0.3)$ & $12.8 \pm 0.2$ & $(13.3 \pm 0.1)$ \\
GHG & $15.6 \pm 0.2$ & $12.1 \pm 0.1$ & $(14.4 \pm 0.2)$ & $9.4 \pm 0.2$ \\
& $(16.5 \pm 0.2)$ & $(14.1 \pm 0.1)$ & $11.9 \pm 0.1$ & $(12.2 \pm 0.2)$ \\
GGH & $14.8 \pm 0.3$ & $11.6 \pm 0.1$ & $(13.4 \pm 0.1)$ & $9.51 \pm 0.3$ \\
& $(15.5 \pm 0.3)$ & $(13.5 \pm 0.1)$ & $18.8 \pm 0.2$ & $(12.3 \pm 0.3)$ \\
GGG & $\mathrm{N} / \mathrm{A}^{2}$ & $19.1 \pm 0.1$ & $(24.8 \pm 0.2)$ & $15.5 \pm 0.2$ \\
& & $(26.0 \pm 0.1)$ & $(24.0 \pm 0.2)$ \\
\hline
\end{tabular}

aValues expressed as percent of maximum CAD voltage, with values corrected for degrees-of-freedom in parenthesis. $\%^{2} U$ nable to obtain comparable dissociation thresholds due to low intensity/absence of complex or multiple dissociation pathways.

because of the competition for retention of the proton by the tripeptide versus 18-crown- 6 .

When dissociating complexes such as these and comparing energies required for a certain degree of fragmentation, the size of the complexes must be considered. Compounds that contain more atoms than others have more degrees of freedom with which to distribute energy, meaning that it requires more energy to break a bond in a larger molecule than is required for an equivalent bond in a smaller molecule, within the same time frame. This kinetic shift problem was compensated by correcting the $10 \%$ dissociation thresholds for the number of atoms in the complex using the following equation:

$$
E(\text { Threshold, Corrected })=E(\text { Threshold }) * \frac{N_{\text {ref }}}{N_{\text {complex }}}
$$

(where $N=3 n-6$ and $n$ is the number of atoms in the complex).

Tables 2, 3, and 4 contain the $10 \%$ dissociation threshold values obtained for the thirteen protonated peptides complexed with the four different polyethers. The thresholds are displayed both as the raw data and, in parentheses, the values corrected for degrees of freedom (the reference was the protonated KGG/18crown-6 complex).

The group of tripeptides studied each contained a single basic amino acid whose position varied, along with two glycine residues. For these small peptides, it was possible to observe the effects of amino acid position and basicity (Table 1) on the relative binding strengths of the polyethers with little contribution from secondary structure. Complexes containing tri-glycine were also analyzed for comparison between side chain and N-terminal protonation. The second set of peptides, (AAAAA, RAAAA, KAAAA, AAAAR), allowed correlation of hydrogen bonding strength with amino acid basicities (N-terminus versus Arg versus Lys), as well as possible effects due to intramolecular hydrogen bonding. The third set consisted of leucine enkephalin (YGGFL) and $\mathrm{Arg}^{3}$-leucine enkephalin (YGRFL). These two were compared to see how the presence of a highly basic amino acid (arginine) affected the binding of the polyether hosts in peptides with bulky amino acid residues. In the peptide without arginine, one would expect protonation and complexation at the N-terminal amine, whereas with the arginine present, there are two possible protonation/complexation sites.

Table 3. Dissociation threshold for protonated penta-alanine peptide complexes ${ }^{\mathrm{a}}$

\begin{tabular}{lcccc}
\hline Peptide & $18 \mathrm{C} 6$ & $15 \mathrm{C} 5$ & Tetraglyme & $12 \mathrm{C} 4$ \\
\hline \hline AAAAA & $16.3 \pm 0.3$ & $13.45 \pm 0.06$ & $13.2 \pm 0.1$ & $9.9 \pm 0.2$ \\
& $(13.7 \pm 0.3)$ & $(12.22 \pm 0.06)$ & $(11.7 \pm 0.1)$ & $(9.8 \pm 0.2)$ \\
KAAAA & $12.7 \pm 0.1$ & $10.1 \pm 0.3$ & $9.7 \pm 0.1$ & $\mathrm{~N} / \mathrm{A}^{\mathrm{b}}$ \\
& $(9.5 \pm 0.1)$ & $(8.1 \pm 0.3)$ & $(7.6 \pm 0.1)$ & $\mathrm{N} / \mathrm{A}^{\mathrm{b}}$ \\
RAAAA & $11.09 \pm 0.01$ & $9.1 \pm 0.1$ & $(7.10 \pm 0.09$ & $\mathrm{N}$ \\
& $(8.19 \pm 0.01)$ & $(7.2 \pm 0.1)$ & $9.6 \pm 0.1$ & $\mathrm{~N} / \mathrm{A}^{\mathrm{b}}$ \\
AAAAR & $11.45 \pm 0.04$ & $9.5 \pm 0.3$ & $(7.4 \pm 0.1)$ & $\mathrm{N} / \mathrm{A}^{\mathrm{b}}$ \\
\hline
\end{tabular}

${ }^{a}$ Values expressed as percent of maximum CAD voltage, with values corrected for degrees-of-freedom in parenthesis.

bUnable to obtain comparable dissociation thresholds due to low intensity/absence of complex or multiple dissociation pathways. 
Table 4. Dissociation threshold for protonated leucine enkephalin peptide complexes ${ }^{\mathrm{a}}$

\begin{tabular}{lcccc}
\hline Peptide & $18 \mathrm{C} 6$ & $15 \mathrm{C} 5$ & Tetraglyme & $12 \mathrm{C} 4$ \\
\hline \hline YGGFL & $15.6 \pm 0.1$ & $12.83 \pm 0.09$ & $12.4 \pm 0.2$ & $9.3 \pm 0.3$ \\
& $(10.4 \pm 0.1)$ & $(9.13 \pm 0.09)$ & $(8.7 \pm 0.2)$ & $(7.0 \pm 0.3)$ \\
YGRFL & $11.6 \pm 0.2$ & $9.6 \pm 0.3$ & $10.1 \pm 0.1$ & $\mathrm{~N} / \mathrm{A}^{\mathrm{b}}$ \\
& $(6.8 \pm 0.2)$ & $(6.0 \pm 0.3)$ & $(6.2 \pm 0.1)$ & $\mathrm{N} / \mathrm{A}^{\mathrm{b}}$ \\
\hline
\end{tabular}

aValues expressed as percent of maximum CAD voltage, with values corrected for degrees-of-freedom in parenthesis.

bUnable to obtain comparable dissociation thresholds due to low intensity/absence of complex or multiple dissociation pathways

An interesting effect of spraying solutions that contain both peptides and polyethers is the stabilization of higher charge states than are accessible with only an acidified peptide solution. In the case of a peptide like RAAAA, where there are two basic sites in close proximity, with differing basicities, it is common to see only a singly charged ion in the mass spectrum, as in Figure 6a. However, upon the addition of 18-crown-6 to the solution, a second proton is stabilized on the peptide, and an intense, doubly protonated, singly ligated species is seen in the mass spectrum, shown in Figure 6b. This multi-protonation effect is due to the charge stabilization provided by the crown ether that solvates the proton on one basic site. Since the first proton (at the lysine side chain) is solvated by 18-crown-6 rather than by intramolecular hydrogen bonding by the peptide, this enables the N-terminus to bind a second proton. Beauchamp and Julian have also reported that the binding of polyether molecules can alter the charge state distribution observed for a given peptide [41].

\section{Sites of Protonation/Complexation and Effect of Peptide Basicity}

Table 1 displays the gas-phase basicities of the tripeptides, with the lysine and histidine side chains being more basic than the N-termini [44]. Thus, if the tripeptides are singly protonated, the proton is expected to be localized on the side chain of the basic amino acid, both in the protonated peptide form and in the protonated polyether/peptide complexes. Only a peptide with no basic side chains, such as GGG, should be protonated on the N-terminal amine.

In Table 2, the dissociation thresholds of the complexes containing the tripeptides and the four polyethers are tabulated. The dissociation thresholds for the tri-glycine/polyether complexes are much higher than those observed for the other complexes. This difference suggests that the complexes involving peptides with basic amino acids have considerably lower hydrogenbonding energies than those complexes involving triglycine, presumably due to the different protonation sites of the peptides ( $\mathrm{N}$-terminus versus side chain) that alter the resulting solvation by the polyethers. Similar conclusions were drawn from the data for the pentapeptide complexes displayed in Table 3. Complexes involving a peptide like AAAAA, which has no basic amino acid side chain, display much higher dissociation thresholds than complexes involving the pentapeptides that contain one basic amino acid.

The gas-phase basicities of the various tripeptides studied were previously reported by Carr and Cassady

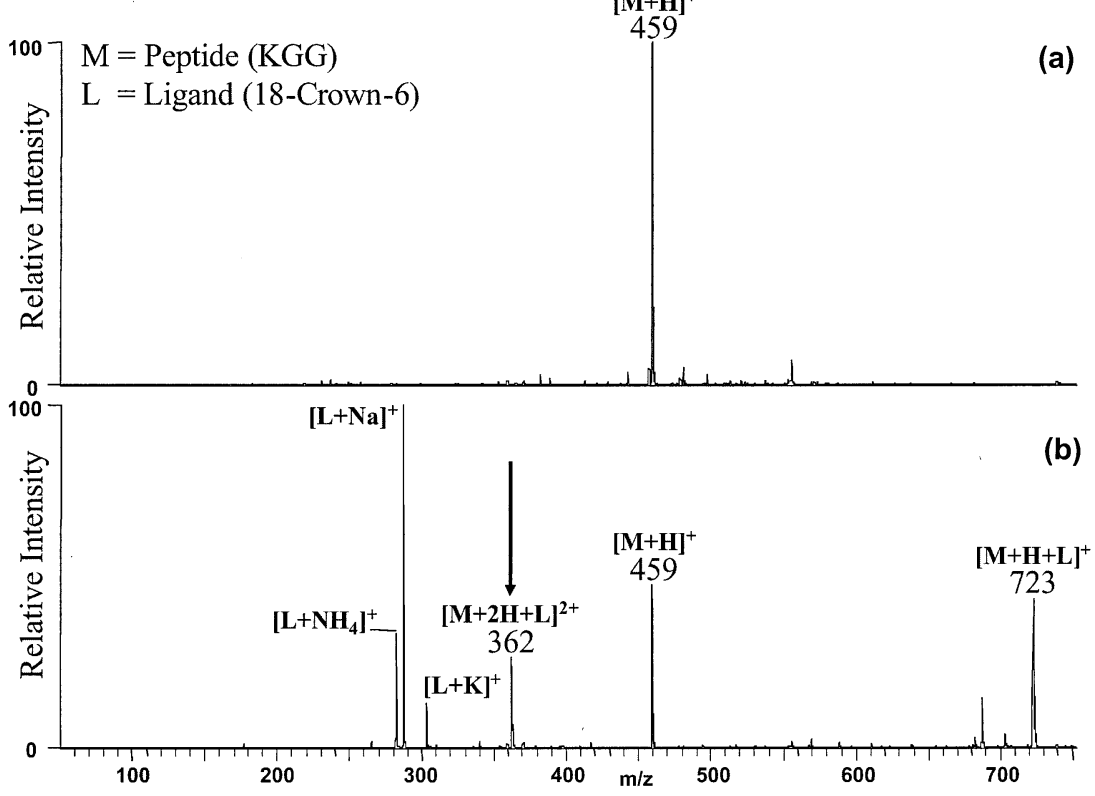

Figure 6. Mass spectra of (a) RAAAA and (b) RAAAA/18-crown-6. 


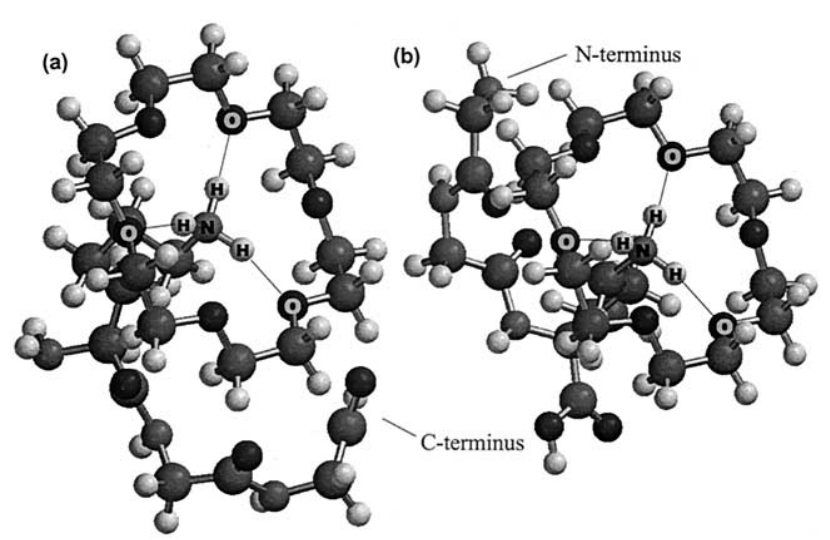

Figure 7. Molecular modeling of the protonated (a) KGG/18crown-6 and (b) GGK/18-crown-6 complexes.

[43], and, after correction to the current NIST scale of gas-phase basicities [44], are listed in Table 1. By comparing the gas-phase basicities of the peptides with the relative dissociation energies of the peptide/polyether complexes, a direct correlation is evident. For instance, in all cases, the dissociation thresholds for complexes containing GGG, the peptide with the lowest gas-phase basicity, were higher than for complexes containing any other peptides. The same type of correlation is seen by comparing the complexes containing lysine tripeptides to those containing histidine tripeptides. The gas-phase basicities of the histidine peptides are lower than those of the analogous lysine tripeptides, and thus the corresponding dissociation thresholds of the complexes containing the histidine tripeptides are higher than those containing the lysine tripeptides. In addition, an inverse relationship is evident between basicity and dissociation threshold amongst the histidine-containing tripeptides. The relative gas-phase basicities are: $\mathrm{HGG}=$ GGH $>$ GHG [43]. The relative dissociation thresholds for the complexes are: GHG > HGG = GGH. As the peptide binds the proton more strongly (as in the case of a more basic peptide), the strengths of the resulting hydrogen bonding interactions between the polyether and proton diminish. This result is consistent with the accepted concept that the strongest hydrogen bonds are the ones in which the protons are most equally shared between two different electron-donating groups [45].

However, in some cases, factors other than gas-phase basicity play a role in the energy required for dissociation. For instance, the gas-phase basicities of the lysinecontaining tripeptides follow the trend: KGG $=$ GGK > GKG [43], and the dissociation thresholds follow the trend: GKG > GGK > KGG. Even though the gas-phase basicities for GGK and KGG are not distinguishable, it requires more energy to dissociate the protonated GGK/polyether complexes than the protonated KGG/ polyether complexes. Examples of the protonated KGG/18-crown-6 and GGK/18-crown-6 complexes, illustrated in Figure 7, show no significant differences in terms of steric interactions between the two peptides and their binding sites, so presumably subtle structural

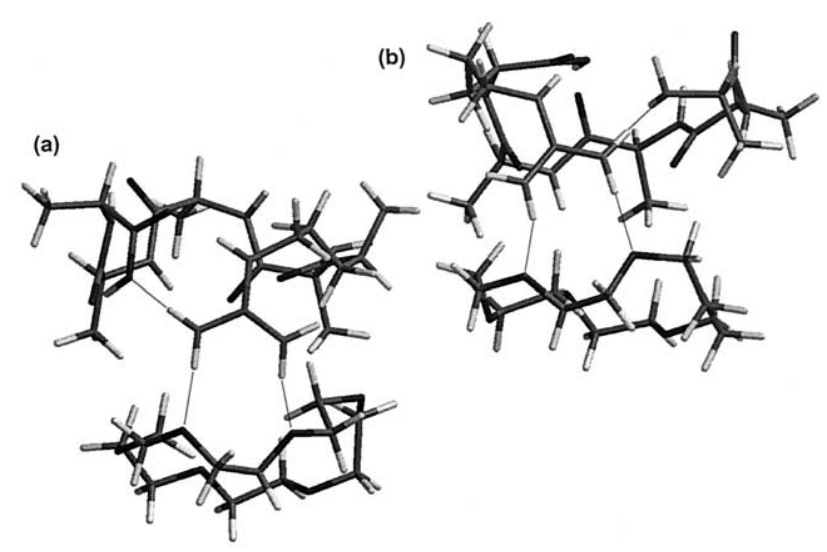

Figure 8. Molecular modeling of the protonated (a) RAAAA/18crown-6 and (b) AAAAR/18-crown-6 complexes.

effects, such as perhaps the interaction of other functional groups (e.g., the carboxyl terminus) with the crown ether ligand, stabilize the GGK complex to a greater extent than the KGG complex. In addition, it is interesting that the threshold measurements involving the noncovalent polyether/peptide complexes allow more sensitive differentiation of KGG and GGK structures than do gas-phase basicity measurements alone. The energy-variable CAD measurements provide an alternative, versatile way to probe subtle differences in structural factors that affect noncovalent interactions.

For all of the protonated pentapeptide/polyether complexes (with the exception of those containing 12crown-4), the dissociation thresholds followed the trend: AAAAA $>$ KAAAA $>$ AAAAR $>$ RAAAA. This trend again correlates with the relative gas-phase basicities of the peptides. Penta-alanine must be protonated and solvated by the polyether at the $\mathrm{N}$-terminus, which is considerably less basic than the lysine or arginine side chains of the other peptides. Thus, the polyethers are able to form more stable hydrogen bonds with protonated AAAAA than with the other pentapeptides, which are protonated at the basic side chains. Lysine has a lower gas-phase basicity than arginine, and this is reflected in the dissociation thresholds of the KAAAA complexes relative to those of the RAAAA and AAAAR complexes, the latter two requiring less energy for dissociation. For the two arginine-containing peptides, the protonated AAAAR/polyether complexes required more energy for dissociation than the protonated RAAAA/polyether complexes. There are two possible explanations for this result; either the position of the arginine residue ( $\mathrm{N}$-terminal versus $\mathrm{C}$-terminal) affects its basicity and therefore the dissociation thresholds, or steric effects and/or intramolecular hydrogen bonding lead to more stable AAAAR complexes, relative to those containing RAAAA. From the molecular models for the AAAAR/18-crown-6 and RAAAA/18-crown-6 complexes (Figure 8), it seems that the steric interactions in these complexes are similar, but the intramolecular hydrogen bonding of the peptides differ. In the RAAAA complex, the arginine undergoes intramolecu- 
lar hydrogen bonding with the carbonyl oxygen of the C-terminal carboxyl group, whereas in the AAAAR complex, the peptide undergoes intramolecular hydrogen bonding with the N-terminal nitrogen. It is likely that these interactions alter the relative basicities of the arginine side chains sufficiently to affect the strengths of the noncovalent interactions with the polyethers. The smallest polyether, 12-crown-4, didn't form a stable complex with any peptide other than AAAAA; consequently, it was not possible to obtain dissociation thresholds. The lack of complex formation was interpreted as an indication that the gas-phase basicities of the peptide and the crown ether were sufficiently different that the crown ether could not form stable hydrogen bonds with the protonated amine of the peptide.

Complexes containing leucine enkephalin (YGGFL) or $\mathrm{arg}^{3}$-leucine enkephalin (YGRFL) with the polyethers were analyzed, and the 10\% dissociation thresholds were obtained for all of the observable complexes. The smallest polyether, 12-crown-4, didn't form a stable complex with YGRFL; thus, it was not possible to obtain a dissociation threshold. Table 4 shows that, for all polyether ligands, the complexes containing leucine enkephalin required more energy for dissociation than the analogous arg $^{3}$-leucine enkephalin complexes. This can be explained by the lower basicity of the Nterminus of YGGFL relative to the more basic arginine side chain of YGRFL, which again results in more balanced and thus stronger hydrogen bonds with the polyether.

\section{Effect of the Gas-Phase Basicity of the Polyethers}

Complexation of protonated peptides was evaluated with four different polyether compounds: 18-crown-6, 15-crown-5, 12-crown-4, and tetraglyme [Tables 2-4]. The three crown ethers were compared to see how the binding strengths of peptides varied as the gas-phase basicity of the crown ether changed. Tetraglyme was used to compare the binding of an acyclic ligand to that of its cyclic analog, 15-crown-5. Regardless of the data set compared, the trend observed is the same in that the threshold values decrease in the order: 18C6 > 15C5/ tetraglyme $>12 \mathrm{C} 4$, a direct correlation with gas-phase basicity of the polyether. This correlation reiterates the importance of the hydrogen-bonding capabilities of each partner in the peptide/polyether complex, in terms of favoring those that can form the most balanced hydrogen bonds and implies that these subtleties can have important cumulative effects in larger biological systems.

\section{Conformational Flexibility of the Polyether}

Tetraglyme and 15 -crown-5 were compared to observe how the conformational flexibility of the polyethers affects binding strength to the peptides. These two polyethers have similar sizes and gas-phase basicities.

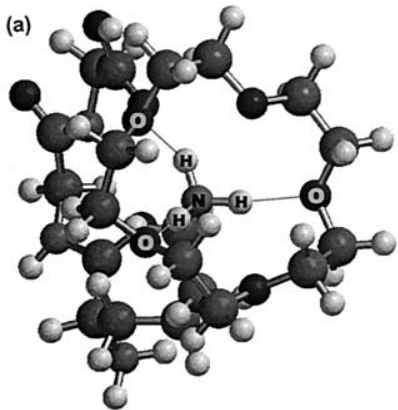

$[\mathrm{KGG}+\mathrm{H}+15-\text { crown-5] }]^{+}$
$[\mathrm{KGG}+\mathrm{H}+\text { tetraglyme }]^{+}$

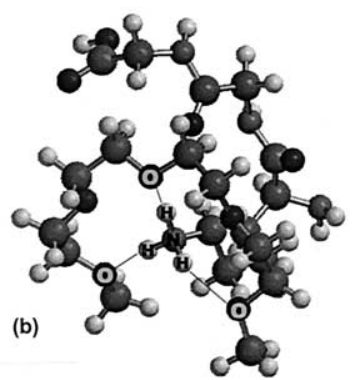

Figure 9. Molecular modeling of the protonated (a) KGG/15crown-5 and (b) KGG/Tetraglyme complexes.

The threshold dissociation data indicates that, for most complexes, 15-crown- 5 binds somewhat more strongly than tetraglyme, except for the protonated GHG complex. As can be observed in Figure 9, the binding to the side chain of lysine in the KGG/polyether complexes results in the formation of similar polyether structures and the same number of hydrogen bonds for both 15-crown-5 and tetraglyme. However, because 15crown-5 is a more rigid, preorganized structure, the hydrogen bonds are slightly stronger, and this is evidenced by the threshold dissociation results. Consequently, the complexes involving lysine-containing tripeptides and 15-crown-5 are more stable and require more energy to dissociate than do the analogous tetraglyme complexes. The only case in which a higher dissociation threshold is observed for a protonated peptide/tetraglyme complex, compared to the analogous 15 -crown-5 complex, is for the complex containing GHG. In fact, molecular models show that the conformational flexibility of the polyether allows the formation of two hydrogen bonds for the tetraglyme complex (Figure 10a). However, in the GHG/15-crown-5 complex, there is only one intermolecular hydrogen bond formed (Figure 10b).
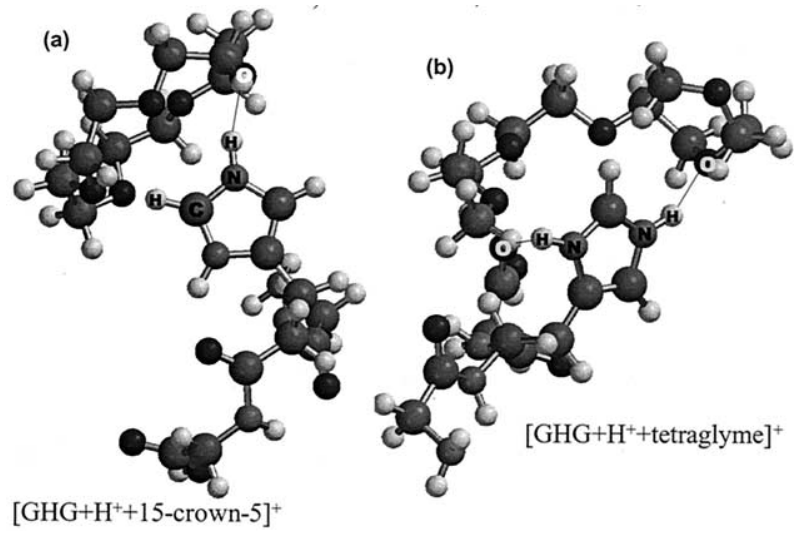

Figure 10. Molecular modeling of the protonated (a) GHG/15crown-5 and (b) GHG/tetraglyme complexes. 


\section{Conclusions}

Based on energy-variable collisionally activated dissociation in a quadrupole ion trap mass spectrometer, the relative strengths of the noncovalent interactions between four polyether molecules with a series of peptides have been evaluated. Three sets of peptide molecules were compared, each providing key information about how amino acid composition and sequence affect their noncovalent interactions with the polyethers. For all three peptide sets, the strengths of noncovalent interactions were inversely proportional to the gasphase basicity of the site of protonation. This was most dramatic when comparing the dissociation thresholds of those peptides with a basic amino acid residue and a comparable peptide in which the most basic site was the $\mathrm{N}$-terminus. In these cases, the peptides protonated at the $\mathrm{N}$-terminus required considerably more energy to dissociate than those protonated at a lysine, histidine, or arginine residue. The binding strengths associated with the various polyethers correlated with their gas-phase basicities. This emphasized the importance of the hydrogen-bonding capabilities of each partner in a peptide/polyether complex, in terms of forming the most balanced (and therefore strongest) hydrogen bonds.

Comparison of the dissociation thresholds of a set of isomeric tripeptides showed that both the position and type of amino acids present affect the strengths of their noncovalent interactions with the polyethers. Relatively subtle differences in structures produced measurable differences in the strengths of noncovalent interactions observed, implying that minor changes in the structures of biological molecules, such as conformational changes, could be revealed by analogous energy-variable CAD measurements. In the case of both lysine and histidine-containing tripeptides, the tripeptides with the structure GXG ( $X=$ lysine or histidine) required more energy for dissociation than the peptides with the XGG or GGX sequences. Comparison of the alaninecontaining pentapeptides made it possible to observe how N-terminal versus C-terminal position of a basic amino acid and the identity of this residue affect the strengths of noncovalent interactions with polyethers.

Comparing the dissociation thresholds obtained for the complexes containing cyclic 15-crown-5 and its acyclic analog, tetraglyme, it is apparent that in most cases 15 -crown- 5 forms more stable complexes. In one case, however, when the flexibility of the acyclic glyme allowed for the formation of more favorable hydrogen bonds than 15-crown-5 did, the tetraglyme complex required more energy for dissociation. This result illustrates the advantage, in some cases, of conformational flexibility over pre-organization.

In summary, the strengths of the noncovalent interactions varied not only with the sequence of the peptide but also its secondary structure. The use of energyvariable CAD gives a sensitive method of evaluating the factors that impact the strengths of noncovalent interactions, and it is envisioned that these measure- ments can be used to probe more complex biological complexes and provide complementary and, in some cases, more sensitive differentiation than provided by $\mathrm{H} / \mathrm{D}$ exchange or gas-phase basicity methods.

\section{Acknowledgments}

The authors gratefully acknowledge the support from the Robert A. Welch Foundation (F-1155).

\section{References}

1. Fenn, J. B., Meng, C. K.; Wong, S. F.; Whitehouse, C. M. Electrospray Ionization for Mass-Spectrometry of Large Biomolecules. Science 1989, 246(4926), 64-71.

2. Karas, M.; Bachmann, D.; Bahr, U.; Hillenkamp, F. MatrixAssisted Ultraviolet-Laser Desorption of Nonvolatile Compounds. Int. J. Mass Spectrom. Ion Processes 1987, 78, 53-68.

3. Veenstra, T. D. Electrospray Ionization Mass Spectrometry in the Study of Biomolecular Noncovalent Interactions. Biophys. Chem. 1999, 79, 63-79.

4. Loo, J. A. Electrospray Ionization Mass Spectrometry: A Technology for Studying Noncovalent Macromolecular Complexes. Int. J. Mass Spectrom. 2000, 200, 175-186.

5. Schalley, C. A. Supramolecular Chemistry Goes Gas Phase: The Mass Spectrometric Examination of Noncovalent Interactions in Host-Guest Chemistry and Molecular Recognition. Int. J. Mass Spectrom. 2002, 194, 11-39.

6. Daniel, J. M.; Friess, S. D.; Rajagopalan, S.; Wendt, S.; Zenobi, R. Quantitative Determination of Noncovalent Binding Interactions Using Soft Ionization Mass Spectrometry. Int. J. Mass Spectrom. 2002, 216, 1-27.

7. Brodbelt, J. S. Probing Molecular Recognition by Mass Spectrometry. Int. J. Mass Spectrom. 2000, 200, 57-69.

8. Pederson, C. J. Cyclic Polyethers and Their Complexes with Metal Salts. J. Am. Chem. Soc. 1967, 89, 2495-2496.

9. Bradshaw, J. S.; Izatt, R. M.; Bordunov, A. V.; Zhu, C. Y.; Hathaway, J. K. Crown Ethers. In Comprehensive Supramolecular Chemistry; Atwood, J. L.; Davies, J. E. D.; Macnicol, D. D.; Vogtle, F., Eds.; Elsevier Science Ltd.: New York, 1996; pp 35-96.

10. Brodbelt, J.; Maleknia, S.; Lagow, R.; Lin, T. Y. Gas-Phase Ion Complexes of a Perfluorinated Cryptand with $\mathrm{O} 2$ and F-. J. Chem. Soc. Chem. Commun. 1991, 23, 1705-1707.

11. Brodbelt, J.; Maleknia, S.; Liou, C. C.; Lagow, R. Selective Formation of Molecular-Oxygen Perfluoro Crown-Ether Adduct Ions in the Gas Phase. J. Am. Chem. Soc. 1991, 113(15), 5913-5914.

12. Maleknia, S.; Brodbelt, J. Gas-Phase Selectivities of Crown Ethers for Alkali-Metal Ion Complexation. J. Am. Chem. Soc. 1992, 114(11), 4295-4298.

13. Maleknia, S.; Brodbelt, J. Cavity-Size-Dependent Dissociation of Crown-Ether Ammonium Ion Complexes in the Gas Phase. J. Am. Chem. Soc. 1993, 115(7), 2837-2843.

14. Liou, C. C.; Brodbelt, J. S. Comparison of Gas-Phase Proton and Ammonium Ion Affinities of Crown Ethers and Related Acyclic Analogs. J. Am. Chem. Soc. 1992, 114(17), 6761-6764.

15. Liou, C. C.; Brodbelt, J. S. Determination of Orders of Relative Alkali-Metal Ion Affinities of Crown Ethers and Acyclic Analogs by the Kinetic Method. J. Am. Soc. Mass Spectrom. 1992, 3(5), 543-548.

16. Wu, H. F.; Brodbelt, J. S. Comparison of the Orders of Gas-Phase Basicities and Ammonium Ion Affinities of Polyethers by the Kinetic Method and Ligand-Exchange Technique. J. Am. Soc. Mass Spectrom. 1993, 4(9), 718-722. 
17. Liou, C. C.; Wu, H. F.; Brodbelt, J. S. Hydrogen-Bonding Interactions in Gas-Phase Polyether Ammonium Ion Complexes. J. Am. Soc. Mass Spectrom. 1994, 5(4), 260-273.

18. Wu, H. F.; Brodbelt, J. S. Gas-Phase Complexation of Monopositive Alkaline-Earth Metal-Ions with Polyethers-Comparison with Alkali-Metal Ion and Aluminum Ion Complexations. J. Am. Chem. Soc. 1994, 116(14), 6418-6426.

19. Wu, H. F.; Brodbelt, J. S. Influence of Heteroatom Donors on the Orders of Relative Gas-Phase Binding Affinities of Macrocyclic Polyethers. J. Inclusion Phenom. Mol. Rec. Chem. 1994, 18(1), 37-44.

20. Alvarez, E. J.; Wu, H. F.; Liou, C. C.; Brodbelt, J. Collisionally Activated Dissociation of Transition Metal Ion/Polyether Complexes in a Quadrupole Ion Trap. J. Am. Chem. Soc. 1996, 118(38), 9131-9138.

21. Colorado, A.; Brodbelt, J. An Empirical Approach to Estimation of Critical Energies by Using a Quadrupole Ion Trap. J. Am. Soc. Mass Spectrom. 1996, 7(11), 1116-1125.

22. Cooks, R. G.; Patrick, J. S.; Kotiaho, T.; McLuckey, S. A. Thermochemical Determinations by the Kinetic Method. Mass Spectrom. Rev. 1994, 13(4), 287-339.

23. Zhang, H.; Chu, J. H.; Leming, S.; Dearden, D. V. Gas-Phase Molecular Recognition-Gas-Phase Crown-Ether Alkali-Metal Ion Complexes and Their Reactions with Neutral Crowns. J. Am. Chem. Soc. 1991, 113(19), 7415-7417.

24. Zhang, H.; Dearden, D. V. The Gas-Phase Macrocyclic EffectReaction-Rates for Crown Ethers and the Corresponding Glymes with Alkali-Metal Cations. J. Am. Chem. Soc. 1992, 114(7), 2754-2755.

25. Chu, I. H.; Zhang, H.; Dearden, D.V. Macrocyclic Chemistry in the Gas-Phase-Intrinsic Cation Affinities and Complexation Rates for Alkali-Metal Cation Complexes of Crown-Ethers and Glymes. J. Am. Chem. Soc. 1993, 115(13), 5736-5744.

26. Dearden, D. V.; Zhang, H.; Chu, I. H.; Wong, P.; Chen, Q. Z. Macrocyclic Chemistry without Solvents-Gas-Phase Reaction-Rates. Pure Applied Chem. 1993, 65(3), 423-428.

27. Wong, S. H.; Antonio, B. J.; Dearden, D. V. Gas-Phase Studies of Valinomycin Alkali-Metal Cation Complexes-Attachment Rates and Cation Affinities. J. Am. Soc. Mass Spectrom. 1994, 5(7), 632-637.

28. Chu, I. H.; Dearden, D. V. Effects of Alkyl Substitution on the Multidentate Attachment of Alkali-Metal Cations by Ligands in the Gas Phase-Kinetics and Thermochemistry of CationBinding by Isomers of Dicyclohexano-18-Crown-6. J. Am. Chem. Soc. 1995, 117(31), 8197-8203.

29. Chen, Q. Z.; Cannell, K.; Nicoll, J.; Dearden, D. V. The macrobicyclic Cryptate Effect in the Gas Phase. J. Am. Chem. Soc. 1996, 118(27), 6335-6344.

30. Wong, S. H.; Yu, X. J.; Dearden, D. V. Complexes of p-TertButylcalix[4]Arene with Mono- and Dipositive Cations in the Gas Phase. Inorg. Chim. Acta 1996, 246((1-2)), 259-265.

31. Kellersberger, K. A.; Dejsupa, C.; Liang, Y. J.; Pope, R. M.; Dearden, D. V. Gas-Phase Studies of Ammonium-Cyclodextrin Compounds Using Fourier Transform Ion Cyclotron Resonance. Int. J. Mass Spectrom. 1999, 193((2-3)), 181-195.

32. More, M. B.; Glendening, E. D.; Ray, D.; Feller, D.; Armentrout, P. B. Cation-Ether Complexes in the Gas Phase: Bond
Dissociation Energies and Equilibrium Structures of $\mathrm{Li}+[\mathrm{O}(\mathrm{CH} 3)(2)](x), x=1-4$. J. Phys. Chem. 1996, 100(5), 1605-1614.

33. Ray, D.; Feller, D.; More, M. B.; Glendening, E. D.; Armentrout, P. B. Cation-Ether Complexes in the Gas Phase: Bond Dissociation Energies and Equilibrium Structures of $\mathrm{Li}+$ (1,2-Dimethoxyethane) $(x), x=1$ and 2 , and $\mathrm{Li}+(12$-crown-4). J. Phys. Chem. 1996, 100(40), 16116-16125.

34. More, M. B.; Ray, D.; Armentrout, B. Cation-Ether Complexes in the Gas Phase: Bond Dissociation Energies of $\mathrm{M}+($ Dimethyl Ether $)(x), x=1-3, \mathrm{M}+(1,2$-Dimethoxyethane $)(x), x=1$ and 2 , and $\mathrm{M}+(12$-crown-4) where $\mathrm{M}=\mathrm{Rb}$ and Cs. J. Phys. Chem. A 1997, 101(37), 7007-7017.

35. More, M. B.; Ray, D.; Armentrout, B. Cation-Ether Complexes in the Gas Phase: Bond Dissociation Energies of $\mathrm{K}+$ (Dimethyl Ether $)(x), x=1-4 ; \mathrm{K}+(1,2$-Dimethoxyethane $)(x), x=1$ and 2, and $\mathrm{K}+$ (12-crown-4). J. Phys. Chem. A 1997, 101(23), $4254-4262$.

36. More, M. B.; Ray, D.; Armentrout, B. Cation-Ether Complexes in the Gas Phase: Bond Dissociation Energies of $\mathrm{Na}+(\mathrm{Di}-$ methyl Ether)( $x), x=1-4 ; \mathrm{Na}+(1,2$-Dimethoxyethane) $(x), x=$ 1 and 2; and Na + (12-crown-4). J. Phys. Chem. A 1997, 101(5), 831-839.

37. More, M. B.; Ray, D.; Armentrout, B. Intrinsic Affinities of Alkali Cations for 15-Crown-5 and 18-Crown-6: Bond Dissociation Energies of Gas-Phase M + Crown Ether Complexes. J. Am. Chem. Soc. 1999, 121(2), 417-423.

38. Armentrout, B. Cation-Ether Complexes in the Gas Phase: Thermodynamic Insight into Molecular Recognition. Int. J. Mass Spectrom. 1999, 193, 227-240.

39. Meot-Ner, M. The Ionic Hydrogen Bond. 3. Multiple NH + ... O and $\mathrm{CH}+\ldots \mathrm{O}$ Bonds. Complexes of Ammonium Ions with Polyethers and Crown Ethers. J. Am. Chem. Soc. 1983, 105, 4912-4915.

40. Cunniff, J. B.; Vouros, P. Mass and Charge Assignment for Electrospray Ions by Crown Ether Adduction. Rapid Commun. Mass Spectrom. 1994, 8, 715-719.

41. Julian, R. R.; Beauchamp, J. L. Site Specific Sequestering and Stabilization of Charge in Peptides by Supramolecular Adduct Formation with 18-Crown-6 Ether by way of Electrospray Ionization. Int. J. Mass Spectrom. 2001, 210/211, 613-623.

42. Julian, R. R.; Akin, M.; May, J. A.; Stoltz, B. M.; Beauchamp, J. L. Molecular Recognition of Arginine in Small Peptides by Supramolecular Complexation with Dibenzo-30-Crown-10 Ether. Int. J. Mass Spectrom. 2002, 220, 87-96.

43. Carr, S. R.; Cassady, C. J. Gas-Phase Basicities of Histidine and Lysine and Their Selected Di- and Tripeptides. J. Am. Soc. Mass Spectrom. 1996, 7, 1203-1210.

44. Hunter, E.; Lias, S. G. NIST Standard Reference Database Number 69; Mallard, W. G.; Lindstromj, J., Eds.; National Institute of Standards and Technology: Gaithersburg, MD, 2003; (http://webbook.nist.gov).

45. Davidson, W. R.; Sunner, J.; Kebarle, P. Hydrogen-Bonding of Water to Onium Ions-Hydration of Substituted Pyridinium Ions and Related Systems. J. Am. Chem. Soc. 1979, 101(7), 1675-1680. 